\title{
ARTICLE
}

\section{Application of Aerosol Formation to Radiation Dosimetry in High-Dose Radiation Fields}

\author{
Naoyuki OSADA ${ }^{1 *}$, Yuichi OKI ${ }^{2}$, Keizou YAMASAKI² and Seiichi SHIBATA ${ }^{2}$, \\ ${ }^{1}$ Department of Energy and Hydrocarbon Chemistry, Graduate School of Engineering, Kyoto University \\ Kyodai-Katsura, Nishikyo-ku, Kyoto, 615-8510, Japan \\ ${ }^{2}$ Research Reactor Institute, Kyoto University, Kumatori-cho, Sen-nan-gun, Osaka, 590-0494, Japan
}

\begin{abstract}
The relationship between the exposure rate and the concentration of the particles was investigated to apply the aerosol formation to radiation dosimetry. To clarify the relationship, an irradiation experiment was carried out at Co-60 gamma-ray irradiation facility. The simple measurement system consisting of transport tubes, an irradiation chamber and aerosol measurement instruments were employed. Both of number concentration and size distribution of the formed aerosol particles in irradiated air were determined using a differential mobility analyzer and a condensation particle counter. The exposure rate was in the range from 1.3 to $22 \mathrm{C} / \mathrm{kg} / \mathrm{h}$. Formed aerosol particles showed log-normal size distributions. The geometric mean diameters were found to be in the range of 6 to $25 \mathrm{~nm}$ depending on the exposure rate. As the exposure rate was increased, both particle size and geometric standard deviation were increased. Similarly to the particle size, the number concentration increased with an increase of the exposure rate. The number concentration and particle size were found to be proportional to the exposure rate. The exposure measurement using aerosol particle counting is, therefore, a promising technique for high-dose radiation fields such as accelerator rooms in machine operation.
\end{abstract}

\section{KEYWORDS: aerosol particle formation, dosimetry, particle size formation, radiolysis of air}

\section{Introduction}

It is known that irradiation to the air causes radiolysis of air molecules and the products form nano-size aerosol particles. It is assumed that number and particle size distribution of the formed aerosol particles are related to exposure and/or exposure rate to the air. The measurement of the aerosol particle would be used in dosimetry at high-exposure rate in accelerator facility. The measurement of aerosol is able to be carried out quickly and real-time. Only an air sampling tube is required in the target place and the formed aerosol is carried through the tube using a pump to measurement station. This method requires no material, such as metals and alanine, is installed to measurement. The aerosol measurement is easier than gas analysis or activity measurement.

The aerosol formed by radiation has been studied. Wood et al. reported beta-ray from tritium formed particles in argon gas including $\mathrm{H}_{2} \mathrm{O}$ vapor, $\mathrm{SO}_{2}$ and ammonia ${ }^{1)}$. Ramamuthi et al. studied alpha-ray emitted from radon-daughter nuclides formed aerosol particles ${ }^{2}$. Keng et al. irradiated benzene and acetylene gas by X-ray and observed the formed particles ${ }^{3)}$. Endo et al. investigated to form particles using neutron beam, however, no particle was detected ${ }^{4}$. These results showed ionization of the air molecules made low-pressure products such as nitrogen oxide and sulfur oxide which formed particles.

*Corresponding Author, E-mail:osada@rri.kyoto-u.ac.jp

(C)2012 Atomic Energy Society of Japan, All Rights Reserved.
In this work, particles formed by irradiation were measured to reveal correlation between exposure rate and property of the particles. This correlation was tested for dosimetry. Number concentration and size distribution of the particles were measured in ${ }^{60} \mathrm{Co}$ irradiation facility. Capability of dosimetry using measurement of aerosol was discussed.

\section{Experimental}

Measurements were carried out in the ${ }^{60} \mathrm{Co}$ gamma-ray irradiation facility in Research Reactor Institute, Kyoto University. Experimental setup was shown in Figure 1. Aerosol-free air was irradiated in chambers installed in the irradiation room. Two chambers (1.7 and 50 litters) were tested to find optimal chamber size. The irradiation chamber was placed at four irradiation points. The exposure rates were $1.3,6.5,8.3$ and $22 \mathrm{C} / \mathrm{kg} / \mathrm{h}$. The exposure rate was measured with alanine dosimeter. Aerosol-free air was prepared using polyethersulphone cartridge filter (ADVANTEC, 0.8 micro-meter pore) from room air. The filtered air was confirmed to be aerosol-free with condensation particle counter (CPC, TSI Inc., Model 3025A). The air was introduced to the chamber set in the irradiation room using silicone tube of 7 -meter length and $1-\mathrm{cm}$ diameter. The temperature of the air was 16 degree Celsius. The relative humidity was $45 \%$. 


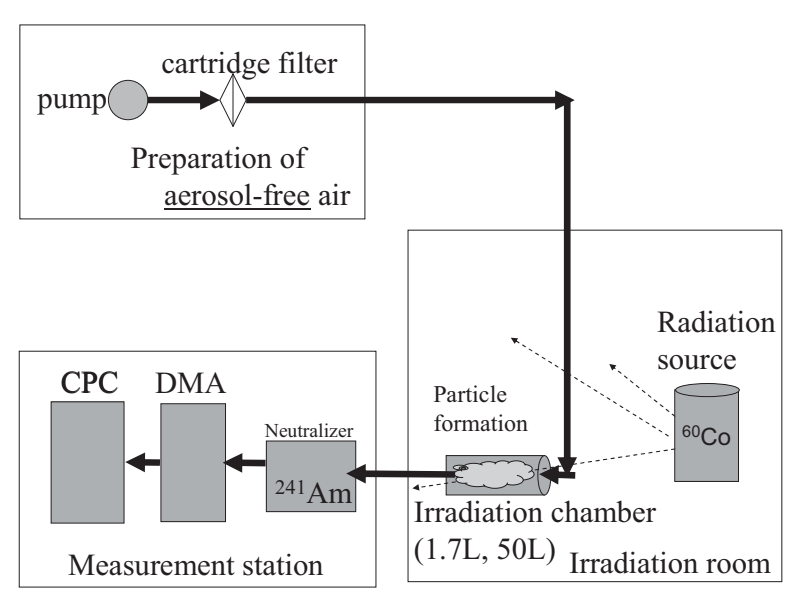

Fig. 1 Schematic diagram of experimental setup.

The irradiated air including the formed particles was transferred to the measurement station using tube. The particles were neutralized by alpha-ray emitted from an ${ }^{241} \mathrm{Am}$ alpha source. The radioactivity was $3.7 \mathrm{MBq}$, which was too weak to form aerosol particles by its alpha-ray radiation. Differential mobility analyzer (DMA, TSI Inc., Model 3070) classified the particles according to size in the range of $3.7-200 \mathrm{~nm}$. The high-voltage of DMA was controlled manually in the range of $0-10000 \mathrm{~V}$. The classified particles were counted by the CPC. The CPC was connected to a $\mathrm{PC}$ to record the numbers of the aerosol particles. Airflow rate which determined the irradiation time in the irradiation chamber was measured with mass flow meters (Horiba STEC, Model SER-21). Flow rates were in the range of 1.5-10.6 litter per minute in the 1.7-litter chamber, in the range of 4-18 litter per minute in the 50-litter chamber. The 1.7-litter chamber was applied to measurement of number concentration and particle size distribution. The 50-litter chamber was applied to measurement of number concentration. The measurement of particle size distribution was performed in 5 minutes.

\section{Results and Discussion}

\section{Particle Size Distributions}

The aerosol particles formed by irradiation had log-normal size distributions. During irradiation, the size distribution was stable. This stability is important for application of aerosol measurement.

Figure 2 shows relationship between the irradiation time and geometric mean diameter (GMD). It was close to the diameter of aerosols formed in a high-energy proton accelerator, high energy accelerator research organization ${ }^{5)}$

The irradiation time was obtained by volume division by flow rate. The results using the 1.7-litter chamber showed linear relationships between the irradiation time and GMD. Higher exposure rate made large particles at the same irradiation time. And long irradiation time grew the particles. The particles grew in the long time irradiation. While the nano-particles acts like molecules, the large partilces acts balls in the air. The grown particles less diffused and gained inertia force. They are supposed to be attached to the wall of the chamber or fell to the bottom.

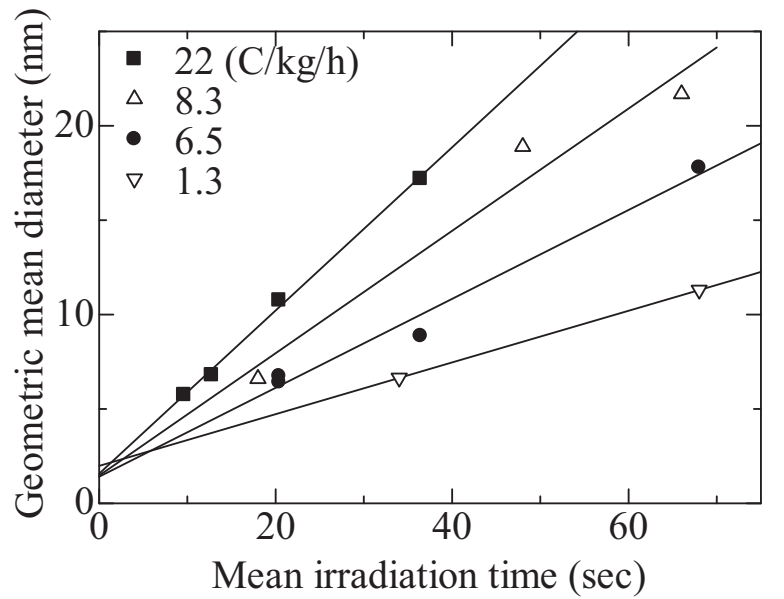

Fig. 2 Relationship between mean irradiation time and geometric mean diameter of the particles in 1.7-litter chamber.

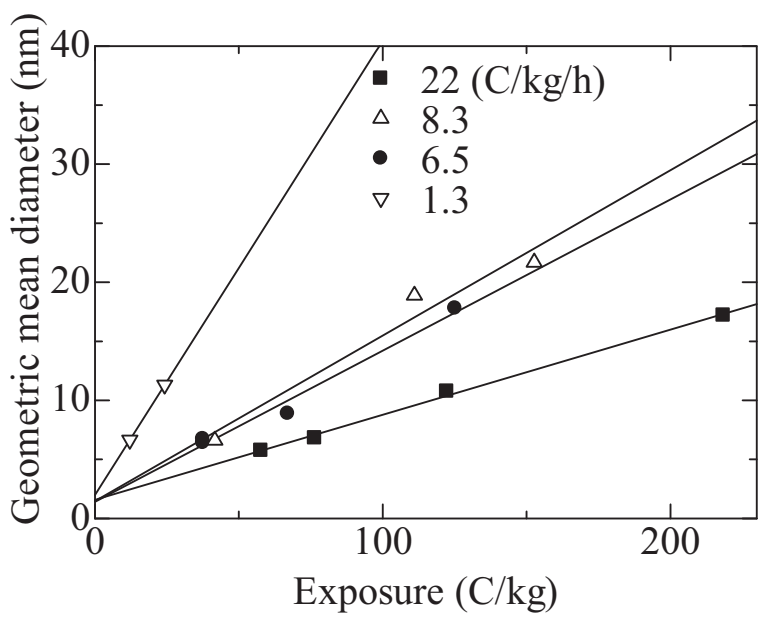

Fig. 3 Relationship between the particle size and exposure in 1.7-litter chamber.

Smaller particles stayed in the air, and larger particles were eliminated in the chamber. The irradiated air in large chamber stayed long time. Too long irradiation time is unsuitable.

Figure 3 shows relationship between absorbed exposure to the air and GMD. Higher exposure resulted in larger particles. At the same exposure, particles formed by lower exposure rate irradiation was larger. Lower exposure rate required long irradiation time to gain the same exposure, which enlarged the particles.

The dependence of the growth rate on the exposure rate was depicted in Figure 4. The growth rate was obtained from Figure 2. The growth rate in the 1.7-litter chamber was expressed as a linear function of the exposure rate. The exposure rate dominated the rate of chemical reactions due to ionaization caused by radiation. The products of the chemical reactions was thought to be condensed by attachment of the particles.

The particle growth was supposed to be caused by coagulation and/or condensation. The particle growth rate was expressed as equation 1. In the condensation process, for particles smaller than gas mean free path, the growth 


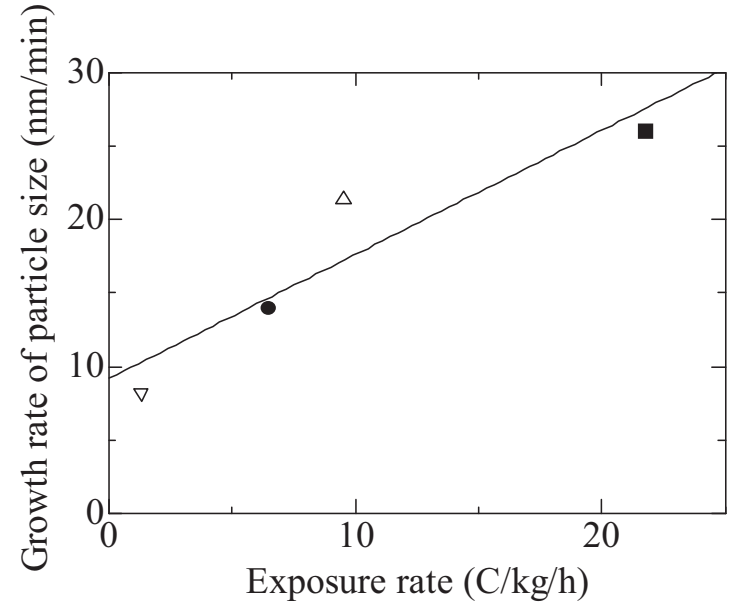

Fig. 4 Growth rate of the particle size in Fig. 2.

rate depends on the rate of random molecular collisions between aerosol particle and gas molecules. The rate of particle growth is

$$
\frac{d\left(d_{p}\right)}{d t}=\frac{2 M \alpha_{c}\left(p_{a}-p_{d}\right)}{\rho_{p} N_{a} \sqrt{2 \pi m k T}}
$$

where $M$ is the molecular weight of the liquid, $\alpha$ is condensation coefficient, $\rho$ is the density of the particle, $p_{a}$ is the ambient partial pressure of vapor, $p_{d}$ is the partial pressure at the particle surface, and $N_{a}$ is Avogadro's number ${ }^{5)}$.

The partial pressure on the particle surface, $p_{d}$ is

$$
p_{d}=p_{s} \exp \left(\frac{4 \gamma M}{\rho R T d}\right)
$$

where $p_{s}$ is vapor pressure for a plane surface, $\gamma$ is the surface tension and $d$ is the particle diameter. The rate of particle growth depends on ambient vapor pressure, $p_{a}$. This growth rate formula shows the dependence on the partial pressure on particle surface. The partial pressure is a function of the exposure rate. In this study, the growth rate is a linear function of the exposure rate (Figure 4). The number concentration of radiolysis aerosol was up to $4 \times 10^{6}$ $\mathrm{p} / \mathrm{cm}^{3}$. Reduction by half of number concentration needs 33 minutes, and doubling particle size needs 4 hours ${ }^{6)}$ at $10^{6}$ particles $/ \mathrm{cm}^{3}$. The concentration of formed aerosol particles is not sufficient to coagulate particles in irradiation time of this experiment, in a few minutes. The growth of particle size was not caused by coagulation but condensation from the gas. It has been considered that the particle formed by irradiation was volatile; it was hard to stay in air. In the case of unstable pure water particle, the vapor pressure on particle surface was calculated (Figure 5). The vapor pressure which has over $10 \mathrm{~nm}$ diameter is approximately $2.7 \mathrm{kPa}$, and it is close to the vapor pressure for a plain surface when the particle size grows. The aerosol formed by irradiation is water mixture and has lower vapor pressure. And it is more stable than a pure water particle. The ambient vapor pressure was not measured. The calculated pressure of nitric acid from the reported $\mathrm{G}$ value $\mathrm{e}^{7)}$ is $10^{-2} \mathrm{~Pa}$ at 8.3 $\mathrm{C} / \mathrm{kg} / \mathrm{h}$ irradiation in $1 \mathrm{~min}$.
In Fig. 4, the line passes through the origin of coordinates. The humidity in the irradiated air was supposed to increase the growth rate.

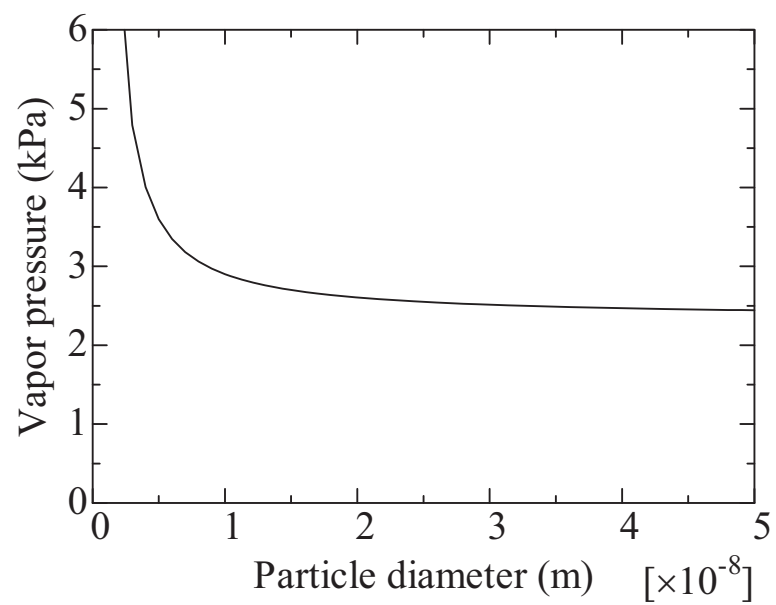

Fig. 5 Calculated vapor pressure on surface of pure water particle using Eq. 2.

\section{Number Concentrations}

Figure 6 shows relationships between particle number concentration and mean irradiation time. The number concentrations were calculated by integrating particle size distributions obtained by the DMA measurement. Similarly to the particle size (Figure 2), the concentration increased with an increase of the irradiation time. In Figure 6, the $\mathrm{x}$-intercept was positive value. This value indicated threshold of particle formation. A certain concentration of gas molecules formed by radiolysis was needed to create the particles. On the other hand, in Figure 2 and Figure 3, the $y$-intercept was positive. The formed particles had minimum particle diameter, around $2 \mathrm{~nm}$. The particle less than the size could not survive long time.

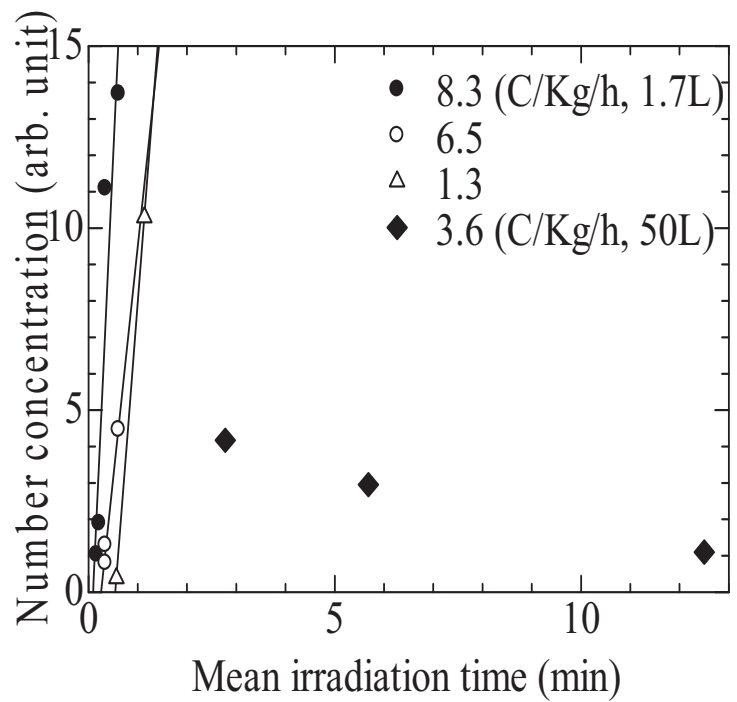

Fig. 6 Relationship between number concentration and mean irradiation time. In 50-litter chamber, formed particles were lost. 
The number concentration in the 1.7-litter chamber was expressed as a linear function of the irradiation time. However, in 50-litter chamber, number concentration was decreased during long irradiation time. Too long time irradiation will result in lost of the particles. In aerosol-free air, the particle formation was occurred by condensation of the formed gas. In circumstance including particles, the gas was consumed for not particle formation but growth of the particles. Once particle was included in the air, the particle formation was hardly happened during machine operation ${ }^{8)}$.

\section{Application of Aerosol Measurement for Dosimetry}

The experiments showed that the relationship between the exposure rate and diameter of formed particles (Figure 3). The growth rate of particle size of formed particles indicated the exposure ratPe. The growth rate was obtained from Figure 2. The particle size did not indicate total exposure to the air. Long time irradiation and low exposure rate made the same size particles formed by short time irradiation and high exposure rate. The number concentration had dependence on the exposure rate and its measurement is easy. However, the number concentration was unstable. Formed particles attached to the wall of the irradiation chamber during they stayed in the chamber. Quick transport and measurement of the particles is needed. The measurement of particle size is able to apply to dosimetry.

\section{Conclusion}

The exposure rate and irradiation time affected the formed particle size. Large particle was formed by long time with high exposure rate irradiation. Only particle size cannot indicate exposure. The exposure rate was obtained from the growth rate of particles. The exposure rate dominated the vapor pressure of formed gas by radiolysis. This relationship is useful for dosimetry at high exposure room such as accelerator facilities.

\section{References}

1) W. P. Wood, A. W. Castleman, Jr. and I. N. Tang, "Mechanisms of Aerosol Formation from $\mathrm{SO}_{2}$ ", J. Aerosol Sci., Vol. 6, 367-374 (1975)

2) M. Ramamurthi, M. Strydom, P.K. Hopke and R.F. Holub, "Nanometer and Ultrafine Particles from Radon Radiolysis", $J$. Aerosol Sci., 24, 393-407 (1993)

3) E.Y. Keng,. "Aerosols produced by X-ray”, J. Coll. Sci. 39 (1), 91-102 (1972)

4) A. Endo, H. Noguchi, Su. Tanaka, Y. Kanda, Y. Oki, T. Iida, K. Sato and S. Tsuda., "Particle Size Analysis of Radioactive Aerosols formed by Irradiation of Argon Using $65 \mathrm{MeV}$ Quasi-monoenergetic Neutrons", Appl. Radiat. Isot., 56, 615-620 (2002)

5) Y. Oki, K. Kondo, Y. Kanda and T. Miura, "Aerosol-size Distribution of Radon Daughter ${ }^{218}$ Po in the Accelerator Tunnel Air", J. Radioanal. Nucl. Chem., Vol. 239, No. 3, 501-505 (1999)

6) William C. Hinds, Aerosol Technology Properties, Behavior, and Measurement of Airborne Particles Second Edition, Wiley-interscience, 1999

7) L. N. Less and A. J. Swallow, "Estimating the Hazard Due to Radiolytic Products from Air", Nucleonics, Vol. 22, No. 9-September, 58-61 (1964)

8) S. Yokoyama, K. Yamasaki, Y. Oki and N. Osada, "Time Variation of Particle Size Distribution of Aerosols Generated at Accelerator Facilities", Hoken Butsuri, 43(4), 333-340 (2008) (in Japanese) 